XXV1.

Aus dem biochemischen Laboratorium (Prof. Dr. M. Jacoby) und dem pathologischen Institut (Geh.- Rat Prof. Dr. C. Benda) des städtischen Krankenhauses Moabit in Berlin.

\title{
Experimentelle und pathologisch-anatomische Untersuchungen an den Nieren bei Vergiftung mit kleinen Gaben Uran.
}

\author{
Von \\ Lasar Dünner und Karl Siegfried.
}

(Mit 2 Abbildungen in Text.)

Grosse Gaben Uran bewirken im Kaninchenversuch wie alle Schwermetallsalze eine schwere typische Glomerulonephritis, die klinisch unter den Erscheinungen der Urämie zum Tode führt. Es ist z. B. Baehr gelungen, die verschiedenen Stufen der entzündlichen Veränderungen an den Nlalpighi'schen Körperchen schrittweise zu beobachten.

Bei weit geringeren Giltmengen $(0,35 \mathrm{mg}$ Urannitrat unter die Haut gespritzt) können Uranpräparate, wie zuerst Pohl berichtet hat, ganz anders geartete Veränderungen in der Funktion der Nieren hervorrufen. Es erfolgt nämlich einige Zeit nach der Vergiftung statt Urämie eine starke Polyurie mit vermehrter Ausschwemmung von $\mathrm{N}$ und $\mathrm{NaCl}$, die bis zum Tode anhält. Die Ursache für diesen tödlichen Verlauf glaubt Pohl in einer spezifischen Nierenschädigung zu sehen: „das Epithel der rewundenen Kanälchen sei grossen Teils fehlend bzw. nekrotisch, die Tunica propria liege bloss; ebenso solle in der Marksubstanz das Kanälehen-Epithel grossen Teils fohlen, bis zu dem Grade, dass ein alveolärer Bau der Niere entstanden sei". Baehr hat diesen Befunden widersprochen; er glaubt zwar ganz ähnliche Bilder wie Pohl gesehen zu haben; jedoch sei $n$ das Epithel an den äusserst erweiterten Kanälchen jederzeit nachweisbar gewesen, wenn auch oft nur als schmaler strukturloser synzytialer Saum".

Vergiftungsversuche Dünner's konnten zwar grundsätzlich die klinischen Angaben Pohl's bestätigen. Er fand, dass die Tiere tatsächlich bei völlig offenen Harnwegen starben; eine ausgesprochene Polyurie wurde nicht immer beobachtet, ebenso keine auffällige Ausschwemmung von Kochsalz. Es ist nun von hoher Bedeutung festzustellen, inwiefern sich die anatomischen Befunde der "Ausschwemmungsnephritis" nach kleinen Urangaben unterscheiden von denen bei Urämie. Uns standen zur Klärung dieser Frage zunächst die Nieren von drei Tieren zur Verfügung 
(263, 276, 265), die Dünner in seiner Arbeit klinisch genau untersucht hatte ${ }^{1}$ ).

I. Die anatomische Untersuchung gestaltete sich folgendermassen: am Morgen nach dem Tode wurden die Tiere seziert, die Nieren nach einfachem Nierenschnitt in 10 proz. Formalin zur Härtung eingelegt. Von einem Verarbeiten der übrigen Organe wurde - leider - Abstand genommen, da sichtbare Veränderungen an ihnen nicht feststellbar waren. Von den Nieren wurden Gefrier- und Paraffinschnitte angefertigt und mit Hämatoxylin-Eosin gefärbt.

Die Befunde bei den einzelnen Tieren geben wir in der Reihenfolge wieder, wie sie Dünner in seiner ersten Arbeit gewählt hat.

Tier Nr. 263 starb 22 Tage nach Einspritzung von 0,35 mg Uran unter die Haut. Bei dem Tiere halten sich die ausgeschiedenen Wasserund Kochsalzmengen in den alten Grenzen wie vor der Vergiftung, während die Stickstoffmengen deutlich zunehmen.

Die Nieren zeigen folgenden Befund: Sämtliche 'l'ubuli enthalten ihr Epithel. Das Epithel ist grossenteils niedrig, die Kanälchen sind nicht übermässig erweitert. Ein kleiner Bruchteil der gewundenen Kanälchen, besonders solcher, die in der Nähe der Glomeruli liegen, zeigt auffallend hohes Epithel, die Zelleiber legen sich, die Lichtung völlig verschliessend, dicht aneinander. Das Protoplasma ist im Verhältnis zu den anderen Kanälchen heller, grauer, träber und deutlich mit Blasen durchsetzt ("vakuolisiert"). Diese Blasen liegen nur nach der Lichtung zu, nie an der Grundfläche der Zelle, erreichen die Grösse der Kerne und überschreiten sie mtiunter auch. Die Gefässschlingen der Malpighi'schen Körperchen sind grossenteils ausgefallen, ein Zeichen, dass wesentliche, besonders produktive Veränderungen an ihnen nicht stattgefunden haben können. Nur ein ganz geringer Bruchteil der Glomeruli zeigt stark zusammengezogene Gefässschlingen, gan\% selten ist der Kapselraum mit geronnener eiweisshaltiger Flüssigkeit gefüllt. In den Schaltstücken liegen sehr zahlreiche schmale hyaline Zylinder, welche die Lichtung des Kanälchens ganz ausfüllen. Diese Zylinder, richtiger bezeichnet diese geronnenen eiweisshaltigen Massen, sind durchgängig vakuolisiert, genau so wie die oben beschriebene Protoplasmazeichnung der Epithelien. Die Blutgefässe an der Uebergangsstelle zwischen Mark und Rinde, auch die der Henle'schen Streifen, sind stark mit Blut gefüllt; besonderer Blutreichtum der Glomerulusschlingen ist nicht feststellbar.

Sehr auffällig ist der Unterschied zwischen verschiedenen Schnitten. Während das oben beschriebene Bild als das gültige angesehen werden muss, gibt es andere, in denen wahrscheinlich infolge eines Kunstfehlers zwischen den Kanälchen schmale, oft gewundene Lücken aufgetreten sind; an ihrer Stelle möchte man leicht das hingehörige Kanälchenepithel für ausgefallen annehmen. Ausserdem finden sich in solchem Schnitte besonders dicht unter der Kapsel viele gewundeve Kanälchen, von deren Epithel nichts weiter übrig geblieben ist als ein schmaler, sehr matt gefärbter,

1) Wir verzichten darauf, an dieser Stelle die klinischen Protokolle einzeln wiederzugeben, sondern verweisen auf die Veröffentlichung Dünner's in der Zeitschrift f. klin. Med. Bd. 81 . Heft 5 u. 6. 
graublauer Saum, der Zellgrenzen nicht mehr erkennen lässt, in welchem aber in regelmäsigen Abständen gut gefärbte Kerne liegen.

Diagnose: rein tubuläre Schädigung der Nieren. Tubulärer Reizungszustand. Tubuläre Entzündung im ersten Beginn.

Tier Nr. 276 lebte auffallend lange; es starb erst 38 Tage nach der Einspritzung von $0,35 \mathrm{mg}$ unter die Haut. In dieser Zeit liessen sich rerschiedene Perioden mit Verminderung und Vermehrung der Stickstoff- und Kochsalzausscheidung nachweisen, die freilich deutlich mit einer Vermehrung der Stickstoffausscheidung abschlossen. Der Nierenbefund ist folgender: Die Epithelien der Kanälchen sind vollständig erhalten. Im Gegensatz zum vorigen Fall sind irgendwelche Unterschiede zwischen einzelnen Schnitten nicht feststellbar. Durch alle Schnitte hin-

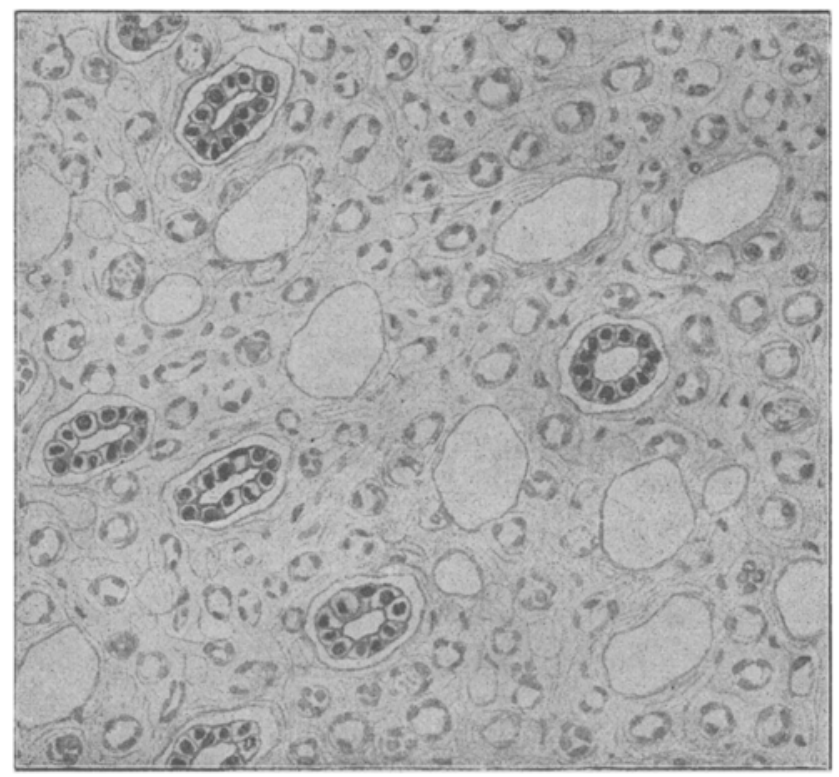

Abbildung 1

durch zeigt das Epithel dieselbe, ein wenig durchlöcherte Beschaffenheit, mit sehr undeutlicher Zeichnung in den Zelleibern. Dies ist wahrscheinlich Leichenerscheinung. Dasselbe gilt von der Löcherigkeit der Zellleiber; denn die Vakulolen sind hier klein, unregelmässig begrenzt, über den ganzen Zelleib verteilt und sind nicht verbunden mit einer Schwellung der ganzen Zelle, wie sie das vorige Präparat zeigte. Nur ganz vereinzelte Kanälchen der Henle'schen Schleifen sind aufs äusserste erweitert, das Epithel ist nur noch als schmaler Saum erkennbar, im Innern liegt frei ein verhältnismässig stark gefärbter liyaliner, leicht gewundener Zylinder. In der Varksubstanz sind die Epithelschläuche der Sammelröhren von ihrer Unterlage vielfach losgelöst, wic durch Kontraktion der Zellen. Solcher Epithelschlauchquerschnitte sind eine ganze Anzahl ausgefallen, so dass die Marksubstanz an diesen Stellen wie nausgepinselt" (Pohl) erscheint (Abb. 1). An den erhaltenen Nachbarn ist dabei deutlich zu 
erkennen, dass das keine natürlichen Veränderungen, sondern Kunstfehler sind. Es stimmt dieser Fehler mit dem oben vom Protoplasma erwähnten überein: die Nieren sind nicht unmittelbar nach dem Tode gehärtet worden. Malpighi'sche Körperchen und Gefässe zeigen keine Veränderungen. Diagnose: Keine wesentlichen krankhaften Veränderungen.

Tier Nr. 265 erkrankte nach dem Typus Pobl, d. h. 11 Tage nach der Vergiftung durch $0,35 \mathrm{mg}$ Uran verschied das Tier unter ausgesprochener Polyurie und Vermehrung der Stickstoff- und Kochsalzmengen. Die auffälligste Veränderung dieser Nieren ist eine äusserste Erweiterung zahlreicher Harnkanälchen, sowohl der gewundenen wie auch der Henle'schen Schleifen; diese sind vielleicht etwas bevorzugt. Solcher erweiterter Kanälchen liegen etwa 8-12 in Gruppen beisammen, und solche Gruppen sind regellos eingestreut zwischen $3-5$ mal so breiten Abschnitten gewöhnlichen Nierengewebes. Vereinzelte Zylinder liegen in den Schaltstücken unter der Oberfläche. Das Epithel der erweiterten Kanälchen

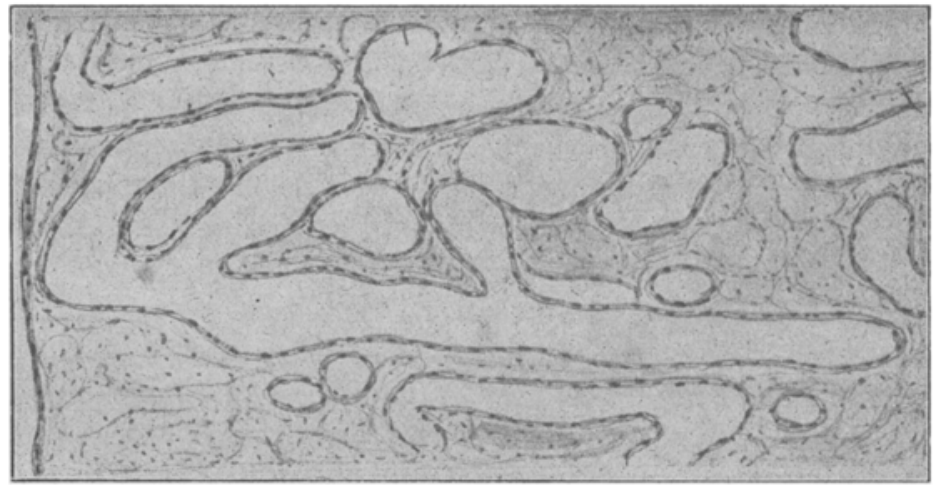

Abbildung 2.

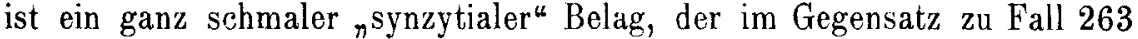
gegen die Lichtung hin seharf begrenzt ist und verbältnismässig dicht gelegene, dunkelgefärbte Kerne enthält (Abb. 2). Häufig ist der Zellsaum von seiner Unterlage abgelöst, springt in die Lichtung vor, so dass er der Gefahr unterworfen ist, auszufallen; dies ist jedoch fast nie geschehen. Dio Kanälchen der übrigen Niere zeigen bei schmaler Lichtung das als Leichenerscheinung beschriebene Aussehen von Fall 276. Nur ein kleiner Bruchteil der Hauptstücke hat gequollene hohe Epithelien, welche die Lichtung prall ausfüllen und in ihren Inneren grössere Hohlräume aufzeigen, eine Veränderung, welche in weit stärkerem Maasse Fall 263 gezeigt hatte.

Von den Glomerulis sind sehr viele ausgefallen. Die Schlingen der erhaltenen sind zum Teil von der Kapsel etwas abgerückt, zeigen aber sonst genau so wenig wie die Gefässe krankhafte Veränderungen.

Diagnose: Rein tubuläre Entzündung leichten Grades.

Epikrise. Die Tiere 263, 276 und 265 sollten der Nachprüfung der Pohl'schen Angaben dienen. Die Veränderungen in allen drei Fällen 
sind im Vergleich zu den von Pohl beschriebenen sehr gering. Dennoch kann man sagen: Die Veränderungen sind durchaus einheitlich, es handelt sich um die verschiedenen Gerade einer rein tubulären Nierenentzündung. Die Schwere der Veränderungen schreitet von 276 zu 263 zu 265 fort. Wichtig ist nunmehr die Feststellung, dass die von Pohl als typisch angegebenen Veränderungen: Ausfall der Epithelien, Blossliegen der Basalmembranen keines der drei Tiere zeigt. Selbst bei dem Tiere 265, das die verhältnismässig grössten Veränderungen aufwies, konnte man trotz stärkster Erweiterung der Kanälchen stets den schmalen Epithelsaum feststellen.

In diesem Widerspruch zu den Pohl'schen Angaben stimmen wir mit Baehr überein, welcher gleichfalls erweiterte Kanälchen sah, jedoch stets an ihnen einen schmalen, mehr oder weniger kernhaltigen Saum nachweisen konnte. Der Unterschied beider Befunde und ihrer Deutungen ist beträchtlich. Pohl glaubt, nach Ausschwemmung ihrer Epithelien könnten die Kanälchen die ihnen normaler Weise obliegende Rückaufsaugung des Harnes nicht vollführen, unmittelbar dureh diese unwiederbringliche tubuläre Schädigung sei die Polyurie, die vermehrte Kochsalzund Stickstoffausscheidung und damit der Tod der Tiere bedingt. In Wirklichkeit aber ist diese Schädigung der Tubuli für den Fortbestand der Niere und damit des Lebens gar nicht crheblich; dic an den Wänden liegenden Epithelien erholen sich rasch, die mehr oder weniger abgestossenen Zelleiber werden mit Hilfe der erhalten gebliebenen Kerne bzw. Zellreste rasch wieder aufgebaut, und Aschoff gibt an, dass solche Nierenentzündung" mit einer völligen Wiederherstellung ausheilen kann.

Eine sehr deutliche Bestätigung dieser Aschoff'schen Vorstellung scheint der Fall 276 zu sein. Das Tier hatte die Einspritzung 38 Tage überlebt. Wir finden an den Nieren keinerlei nennenswerte Veränderungen mehr. Nur ganz spärliche Kanälehen zeigen noch Ansätze zur Erweiterung wie diejenigen Tiere, welche 20-30 'Tage früher gestorben waren. Mau geht wohl nicht fehl anzunehmen, dass hier die Reste einer Veränderung vorliegen, welche ursprünglich ebenso umfangreich war wic bei den anderen Tieren. Bei diesen wurden die Veränderungen im anatomischen Präparat auf der Höhe festgehalten, weil die Tiere schon 12 bzw. 22 Tage nach der Einspritzung eingingen. In 38 Tagen jedoch war die Schädigung der Kanälchen wieder ausgeheilt mit einer fast völligen Wiederherstellung. Dass das Tier 276 überhaupt gestorben ist, kann durch den Befund an den Nieren in keiner Weise begründet werden. Fs wird darüber weiter unten verhandelt werden.

Mit der Annahme der Ausheilung stimmt die Tatsache überein, dass die von Pohl gespritzten Tiere durchschnittlich nur 12 (10-14 Tage) gelebt haben. 11 Tage nach der Einspritzung haben auch wir die gröbsten Veränderungen (im Fall 265) gesehen. Pohl hat also nur die groben Veränderungen beobachten können, hhm mussten daher die von uns aufgedeckten Zusammenhänge verborgen bleiben mit den, wie sich weiter unten zeigen wird, weittragenden Folgerungen. Dadurch, dass Pohl nur grobe Veränderungen gesehen hat, erklärt sich auch die einseitige Deu- 
tung seiner Bilder. Pohl hat genau das gleiche Bild gesehen, wie unser Tier 265 zeigte; massenhaft auls äusserste erweiterte Kanälchen, gewundene wie Henle'sche Schleifen. Bei schwacher Vergrösserung ist ein Epithel überhaupt nicht zu sehen, und auch bei starker Vergrösserung sieht man sehr oft nicht mehr als einen schmalen, an ein Endothel erinnernden Saum. Hierzu kommt die Möglichkeit, dass der Epithelsaum in Pohl's Präparaten tatsächlich nicht mehr vorhanden war. Denn wie wir in Fall 265 und 263 ausdrücklich erwähnt haben, hebt sich das Epithel als schmaler Saum (in 265 zuweilen, in 263 oft) von der Unterlage ab und kann dann ausfallen. Dies ist vielleicht in den Pohl'schen Präparaten geschehen. Ja für das Nierenmark kann man sagen, dass schon Schnitte normaler Nieren bei nicht ganz vorsichtiger Behandlung das Epithel vieler Sammelröhren verlieren. Das zweite Bild von Pohl, das einem Markkegelquerschnitt entstammt, würde einem solchen "normalen" Bilde entsprechen. Dass die Pohl'sehen Bilder wahrscheinlich durch solch einen ihm unbewussten Kunstfehler zustande gekommen sind, zeigt die Abb. 1. Sie entspricht ganz der PohIschen, weist im Gegensatz zu seiner nur noch vereinzelte, erhalten gebliebene Epithelschlauchinhalte auf; sie zeigt nicht die geringste krankhafte Veränderung.

Die anatomische Nachprüfung der Pohl'schen Versuche ergibt demnach: die Einspritzung von $0,35 \mathrm{mg}$ Uran erzeugt beim Kaninchen eine krankhafte Veränderung an den Nieren, welche ausschliesslich die Tubuli betrifft, Hauptstücke und gewundene Kanälchen einerseits (a), Henle'sche Schleifen (b) andererseits.

Die Veränderungen an den Hauptstücken $(a)$ bestehen in einer Schwellung und Vakuolisierung der Protoplasmaleiber, die Auftreibung verlegt die Lichtung, die Kerne rücken an die Basalmembran; die geschwollenen Protoplasmaleiber werden abgestossen, verbacken miteinander und werden als Zylinder fortgeführt, so dass vom Epithel nur noch der basale den Kern enthaltende Abschnitte übrig bleibt. Diese Zylinderbildung bedarf in ihrer Beziehung zum Zustandekommen der charakteristischen Kanälchenerweiterung in den abwärtigen Abschnitten $(b)$ einer besonderen Besprechung.

Dass die Zylinder hauptsächlich (vielleicht alle?) gerade in den Hauptstücken und gewundenen Kanälchen erster Ordnung (a) gebildet werden, kann nicht bezweifelt werden. Wir sehen in vielen Präparaten alle Uebergänge zwischen einem „Exsudat", das die schmale Lichtung innerhalb der vakuolisierten aufgetriebenen Zelleiber prall erfüllt, und vakuoligen eben abgrenzbaren Zylindern und deutlich gleichförmigen Zylindern bei ganz niedrigen Zellresten. Nur hier füllt ein Zylinder die Lichtung des Harnkanälchens vollständig aus. In allen tieferen Abschnitten (b) des Kanälchensystemes ist das Vorhandensein eines Zylinders von einer mehr oder weniger erheblichen Erweiterung des Harnkanälchens begleitet. Dieser Tatsache entspricht, dass die Farbe der Zylinder in den Hauptstücken hell ist, eine dunklere Färbung, das Zeichen des Aelterseins, ist stets erst in den weiter abwärts gelegenen Abschnitten der Kanälchen zu sehen. In diesen kann also die Bildungsstelle der 
Zylinder nicht sein. Das gilt mit voller Sicherheit auch für die gewundenen Kanälchen zweiter Ordnung bis zu ihrer Einmündung in die Sammelröhren; wir haben nämlich in einem Schnitt zwei stark erweiterte Kanälchen in ein gemeinsames erweitertes Rohr einmünden sehen. Auf Grund dieses Bildes (Abb. 2) kann ausgeschlossen werden, was Baehr behauptet, dass die Kanälchenerweiterung mechanisch bedingt sei, d. h. ein im tieferen Abschnitt festgefahrener, die Lichtung verstopfender Zylinder hemme den Abfluss im Kanälehen und bedinge dadurch dessen Erweiterung. Man muss vielmehr annehmen, der Zylinder, der fraglos körperfremde Stoffe enthält, wirke etwa als "negativ chemotaktischer" Reiz auf die Epithelien, an denen er vorbeifliesst; die Zellen antworten auf diesen Reiz mit möglichster Entfernung ihrer Zelleiber. Die Erweiterung der Kanälchen (b) scheint demnach nicht ein passiver, sondern ein aktiver Vorgang zu sein. Diese mit Zylinderaussohwemmung Hand in Hand gehende Erweiterung der Kanälchen befindet sich nach unseren und den Pohl'schen Versuchen auf der Höhe etwa nach 8-20 Tagen, nach 40 Tagen war sie mehr oder weniger beendet.

Eine A useinandersetzung, ob diese Veränderung an den Tubuli überhaupt als Entzündung, als -itis zu bezeichnen sei, scheint unfruchtbar. Sicher kann man sie eine Nierenreizung nennen. Wir glauben uns A sch of $\mathrm{f}$ anschliessen zu dürfen, wenn wir die tatsä̈hlich nachgewiesene Schwellung, Vakuolisierung und Nekrose der Epithelien einerseits, die Exsudatbildung und die Produktion bzw. den Ersatz der zugrunde gegangenen Zelleiber andererseits als Entzündung ansprechen.

Die Pohl'sche Uranvergiftung des Kaninchens mit sehr kleinen Gaben von $0,35 \mathrm{mg}$ Uran auf $1,5-2 \mathrm{~kg}$ Tier ruft an der Niere eine gutartige, rein tubuläre Nephritis hervor, welche mit völliger Wiederherstellung spätestens nach 40 Tagen wieder ansgeheilt ist.

Wenn man nun die anatomischen Befunde in Beziehung $z u$ den klinischen Angaben setzen will, so kann man von vornherein sagen, dass die Tiere nicht an der Nierenveränderung als solcher, wie Pohl angenommen hatte, zugrunde gegangen sind. Denn Pohl hatte die Polyurie und Ausschwemmung von Stickstoff gerade anf den Ausfall des Tubulusapparates zurückgeführt. Dass dies nicht der Fall zu sein braucht, beweist mit Sicherheit das Tier 276, das zwar die klinischen Symptome Pohl's darbot, bei dem aber der Nierenbefund fast normal war. Gerade diese Tatsache muss die Frage anregen, ob nicht das ganze Krankheitsbild als das Zeichen einer Allgemeinvorgiftuug durch das Uran aufzufassen sei. Wir wollen damit keineswegs leugnen, dass bei den Urantieren auch eine Nierenschädigung bestehe; aber sie wären dann Jediglich als Teil im Kreise der Vergiftung aller Organe aufzufassen. Zu dieser Betrachtungsweise zwingt ja schon allein die Ausscheidung von Eiweiss, zum Teil auch von Blut und Zylindern im Urin. Es wäre freilich von Wert zu wissen, warum die verschiedenen Tiere auf die gleichen Gaben Uran verschieden antworteten. Da die Tiere ungefähr gleich schwer waren und mit denselben Mengen vom gleichen Präparat gespritzt wurden, so kann man für den verschiedenen Krankheitsverlauf 
nur eine besondere Widerstandsfähigkeit (276) bzw. Empfindlichkeit dem Uran gegenüber annehmen. Diesen Standpunkt hat bereits Baehr in seinen Versuchen, die den unserigen in vielen Punkten ähneln, vertreten. Er sah bei der gleichen Versuchsanordnung unter 10 Tieren eines von so besonderer Empfindlichkeit, dass es auf Einspritzung von $0,35 \mathrm{mg}$ Uran sogar mit einer Glomerulonephritis antwortete.

II. Für uns ist es nun wesentlich zu wissen, woher die Vermehrung des Wassers, des Stickstoffs und des Kochsalzes stammt. Es besteht, nachdem wir die Erkrankung der Niere als Grund abgelehnt haben; zunächst die Möglichkeit, dass die vermehrte Ausscheidung durch eine Vergiftung des übrigen Körpers bedingt sei; dazu würde die Möglichkeit hinzukommen, dass das Uran spezifisch eine erhöhte Tätigkeit der Niere anrege. Man kann nun diese Frage lösen, wenn man versucht, das Uran nur der Niere einzuverleiben, wobei man die Vorstellung hat, dass das Uran in der Niere haften blcibt und nicht in die übrigen Organe weitergetragen wird. Derartige Versuche sind durchführbar. Denn Baehr konnte mit entsprechenden Gaben, die or in die Arterie einer Niere einspritzte, an dieser eine Glomerulonephritis erzeugen, während an der anderen Niere nur die Tubuli leichte Veränderungen zeigten. Das beweist also, dass der grösste Tejl des Urans tatsächlich in den gespritzten Nieren verblieb. Es gelangten nur ganz geringe Mengen in den Körperkreislauf, die die Malpighi'schen Körper unversehrt lassen und nur die Tubuli angreifen. In diesem Sinne fielen auch eigene Versuche aus, bei denen wir $1 \frac{1}{2}$ bzw. 2 Stunden nach Einspritzen von 0,02 bzw. $0,2 \mathrm{mg}$ Uran in eine Nierenarterie die betreffende Niere operativ entfernten und die Tiere ohne Schädigung der erhaltenen Niere weiterlebten. Nach diesen Erfahrungen haben wir nur sehr kleine Mengen Urannitrat, nämlich $0,042 \mathrm{mg}$, in jede Niercnarterie eingespritzt (Tior 287) und bei einem weiteren Tiere (261) die noch kleinere Menge von 0,013 mg. Wegen der Bedeutung, die gerade die Ergebnisse dieser Versuche zu haben scheinen, lassen wir auch die klinischen Protokolle hier noch einmal folgen (siehe umstehend).

Das Tier 287 hat die Einspritzung 16 Tage überlebt. Es hat ausreichende, nicht vermehrte Urinmengen geliefert, eine auffallende Ausschwemmung von Stickstoff und Kochsalz ist nicht erfolgt; immerhin kann man sagen, dass das Tier bei offenen Harnwegen zugrunde gegangen ist. Der anatomische Befund an den Nieren war folgender: Abgesehen von einem kleinen Infarkt in der einen Niere, der durch die Einspritzung in die Nierenarterio bedingt ist, zeigen die Kanälchen keine nennenswerten krankhaften Veränderungen. Die Epithelien sehen vakuolisiert, aber nicht geschwollen aus, genau so, wie es im Fall 265 als normale Leichenerscheinung beschrieben worden ist. Nur ganz vereinzelte aufsteigende Schenkel Henle'scher Schleilen sind erweitert, haben dabei z. T. einen hyalinen Zylinder frei in sich liegen; auch einige Schaltstücke tragen ausfüllende hyaline Zylinder. Sehr auffällig ist nun das Verhalten der Glomeruli. Im Vordergrunde steht, dass keine einzige Gefässschlinge ausgefallen ist; dass hierfür eine bessere Behandlung der Nieren verantwortlich sei, ist nicht anzunehmen, da die Epithelien der Kanälchen 
Tier Nr. 287.

\begin{tabular}{|c|c|c|c|c|c|c|c|c|c|c|}
\hline \multirow[b]{2}{*}{ Datum } & \multirow{2}{*}{$\begin{array}{c}\mathrm{Ge}- \\
\text { wicht }\end{array}$} & \multirow[b]{2}{*}{ Hafer } & \multirow[b]{2}{*}{ Wasser } & \multicolumn{5}{|c|}{ Urin } & \multirow[b]{2}{*}{$\mathrm{NaCl}$} & \multirow[b]{2}{*}{$\mathrm{N}$} \\
\hline & & & & Menge & $\begin{array}{l}\text { Spez. } \\
\text { Gew. }\end{array}$ & Reakt. & $\begin{array}{c}\text { Alb.u. } \\
\text { Zyl. }\end{array}$ & Sang. & & \\
\hline 27. 6 . & 2530 & 80 & 90 & & & & & & 0,133 & 0,297 \\
\hline 28.6 . & 2430 & 90 & 90 & 74 & 1025 & s. & - & & 0,133 & 0,297 \\
\hline 29.6 . & 2400 & 70 & 90 & 104 & 1025 & s. & - & - & 0,457 & 3,057 \\
\hline 30.6 . & 2450 & 60 & 90 & 34 & 1015 & s. & - & - & 0,074 & 0,778 \\
\hline 1. 7. & 2400 & 10 & 90 & 120 & 1030 & $\mathrm{~s}$ & $-\pi$ & & 0,108 & 1,218 \\
\hline \multirow[t]{2}{*}{ 2. 7.} & 2320 & 90 & 90 & 120 & 1050 & s. & - & - & 0,108 & 1,218 \\
\hline & \multicolumn{3}{|c|}{$\begin{array}{l}\text { Operation + In iekt. v. } 0,042 \mathrm{mb} \\
\text { Urannitrat in jede Nierenarterie }\end{array}$} & & & & & & & \\
\hline 3.7. & 2870 & 90 & 90 & 84 & 1025 & s. & + & - & 0,184 & 1,922 \\
\hline 4.7. & 2380 & 50 & 80 & 50 & 1025 & s. & + & 一 & 0,090 & 1,081 \\
\hline 5.7. & 2370 & 60 & 90 & 80 & 1035 & s. & + & - & 0,320 & 3,964 \\
\hline 6.7. & 2200 & 10 & 90 & 44 & 1045 & s. & + & - & 0,132 & 1,814 \\
\hline 7. 7. & 2050 & 20 & 90 & 68 & 1040 & s. & + & - & 0,204 & 2,189 \\
\hline $8 . ?$ & 1890 & 15 & 90 & 78 & 10 & s. & + & - & 0,218 & 1,850 \\
\hline 9.7. & 19.50 & 70 & 90 & 65 & 1028 & s. & + & - & 0,104 & 1.378 \\
\hline 10.7. & 1890 & 60 & 90 & 84 & 1040 & s. & + & - & 0,151 & 2,414 \\
\hline 11. 7. & $18: 30$ & 40 & 90 & 34 & 1045 & s. & + & - & 0,041 & 1,142 \\
\hline 12.7 & 1700 & 10 & 90 & 88 & 1040 & s. & + & - & 0,123 & 2,654 \\
\hline 13. 7 & 1610 & 55 & 90 & 62 & 1040 & s. & + & - & 0,235 & 2,061 \\
\hline 14.7. & 15 & 40 & 90 & 68 & 1040 & s. & + & - & $0 . \% 31$ & 2.299 \\
\hline 15. 7. & 1570 & 40 & 90 & 84 & $10+0$ & s. & + & + & 0,033 & 1,728 \\
\hline 167. & 1550 & 0 & 90 & 134 & & & + & + & 0,134 & 1.904 \\
\hline 17. 7. & 1380 & 20 & 90 & & & & & & 0,134 & 1,904 \\
\hline 18. 7. & Morg & $s$ tot & den & & & & & & & \\
\hline
\end{tabular}

Tier Nr. 261.

\begin{tabular}{|c|c|c|c|c|c|c|c|c|c|}
\hline \multirow[b]{2}{*}{ Datum } & \multirow[b]{2}{*}{ Gewicht } & \multirow[b]{2}{*}{ Rüben } & \multicolumn{5}{|c|}{ Urin } & \multirow[b]{2}{*}{$\mathrm{NaCl}$} & \multirow[b]{2}{*}{$\mathrm{N}$} \\
\hline & & & Menge & $\begin{array}{c}\text { Spez. } \\
\text { G.'wicht }\end{array}$ & Reakt. & $\begin{array}{c}\text { Alb.u. } \\
\text { Zyl. }\end{array}$ & Sang. & & \\
\hline $\begin{aligned} & 1.1 \\
& 2.1 \\
& 3.1 \\
& 4.1 . \\
& 5.1 \\
& 6.1 \\
& 7.1 \\
& 8.1 \\
& 9.1 \\
& 10.1 \\
& 11.1 .\end{aligned}$ & $\begin{array}{l}2080 \\
2110 \\
2115 \\
2130 \\
2145 \\
2155 \\
2175 \\
2175 \\
2160 \\
2150 \\
2145\end{array}$ & $\begin{array}{l}125 \\
125 \\
125 \\
125 \\
125 \\
125 \\
125 \\
125 \\
125 \\
125 \\
0\end{array}$ & $\begin{array}{l}200 \\
170 \\
115 \\
182 \\
112 \\
184 \\
120 \\
115 \\
132\end{array}$ & $\begin{array}{l}10: 5 \\
1030 \\
1020 \\
1025 \\
1025 \\
1025 \\
1027 \\
1025 \\
1030\end{array}$ & $\begin{array}{l}\text { s. } \\
\text { s. } \\
\text { s. } \\
\text { s. } \\
\text { s. } \\
\text { s. } \\
\text { s. } \\
\text { s. } \\
\text { s. }\end{array}$ & $\begin{array}{l}- \\
- \\
- \\
- \\
= \\
- \\
-\end{array}$ & $\begin{array}{l}- \\
- \\
- \\
- \\
- \\
- \\
-\end{array}$ & $\begin{array}{l}0,990 \\
0,476 \\
0,322 \\
0,327 \\
0,3: 7 \\
0,246 \\
0,809 \\
0,648 \\
0,138 \\
0,237\end{array}$ & $\begin{array}{l}1,019 \\
0,856 \\
0,450 \\
0,301 \\
0.301 \\
0,418 \\
0,797 \\
0,594 \\
0,492 \\
0,646\end{array}$ \\
\hline $\begin{array}{l}12.1 . \\
131 . \\
14.1 . \\
15.1 . \\
16.1 . \\
17.1 . \\
18.1 . \\
19.1 . \\
20.1 . \\
21.1 .\end{array}$ & $\begin{array}{c}\text { Operat. }+ \text { It } \\
1950 \\
1800 \\
1750 \\
1650 \\
1550 \\
1450 \\
1370 \\
1300 \\
1220 \\
1200\end{array}$ & $\begin{array}{l}\text { von } \\
0 \\
0 \\
0 \\
0 \\
0 \\
0 \\
0 \\
0 \\
0 \\
0\end{array}$ & $\begin{array}{l}55 \\
48 \\
\\
82\end{array}$ & $\begin{array}{l}1045 \\
1045 \\
1040 \\
1060\end{array}$ & $\begin{array}{l}\text { S. } \\
\text { s. }\end{array}$ & $\begin{array}{l}+ \\
+ \\
+ \\
+\end{array}$ & $\bar{z}$ & $\begin{array}{l}11.1 \text {. ve } \\
0,085 \\
0,085 \\
0,085 \\
0,462 \\
0,393 \\
0,147 \\
0,147 \\
0,147 \\
0,064 \\
0,064\end{array}$ & $\begin{array}{c}\text { ngegange } \\
0,436 \\
0,436 \\
0,436 \\
1,940 \\
1,842 \\
1,476 \\
1,476 \\
1,476 \\
1,981 \\
1,981\end{array}$ \\
\hline
\end{tabular}


nicht besser erhalten sind als in den übrigen Präparaten, von denen das Ausfallen der Schlingen als Regel beschrieben wurde. Es muss also eine ungewöhnliche Bindung der Schlingen an die Kapsel bestehen. In der Tat findet sich dort, wo zwischen Kapsel und Schlinge überhaupt ein Hohlraum besteht, dieser ausgefüllt mit teils fädigem, teils gekörntem deutlich sichtbarem Exsudat. In der Regel liegen die Schlingen mit dem grössten Teile ihres Umfanges der Kapsel dicht an. Hier besteht deutlich Vermehrung der Kerne und in einem kleinen Teile Zellvermehrung nach dem bekannten Typus der Halbmondbildung. Diese Zellwucherungen springen zuweilen wie ein Polyp in die abführenden Harnkanälchen vor. Vereinzelte Knäuel sind bereits geschrumpft, enthalten jedoch völlig regelrechte Kerne. Es handelt sich demnach um eine Exsudatbildung und produktive Entzündung der Malpighi'schen Körperchen.

Diagnose: Glomerulonephritis geringen Grades.

Das Tier 261 hat 10 Tage nach der Einspritzung von $0,013 \mathrm{mg}$ in jede Nierenarterie gelebt. Nach der Operation hat es keinerlei Nahrung mehr zu sich genommen; deshalb sind die von ihm ausgeschiedenen Urinmengen als verhältnismässig gross zu betrachten, die Stickstoffausscheidung und die des kochsalzes jedoch nicht. Anatomischer Befund: An diesen Nieren ist, wahrscheinlich infolge zu raschen Einspritzens, eine "Perinephritis purulenta" aufgetreten. Die Nieren sind grossenteils nekrotisch. Trotzden ist die Kernfärbung in den Glomerulis erhalten, angedeutet in vielen schmalen aufsteigenden Kanälchen, geschwunden in allen übrigen Kanälchen. In den anderen Abschnitten der Niere sind von den Malpighi'schen Körperchen, deren auch in diesem Falle kaum eines ausgefallen ist, viele vergrössert, ihr Kapselraum ist deutlich erweitert und ausgefüllt durch einen homogenen, blassrosa gefärbten Inhalt ("Exsudat").

Diagnose: Abgesehen von der Perinephritis purulenta frische, leichteste Entzündung an den Malpighi'schen Körperchen (sogenannte Glomerulitis exsudativa).

Wenn auch die beiden Tiere 287 und 261 klinisch einen verschiedenen Verlauf genommen haben, so scheint es uns doch gerechtfertigt, sie gemeinsam epikritisch zu betrachten. Dazu berechtigt uns die Tatsache, dass wir bei Einspritzung unter die Haut bei drei Tieren gleichfalls beträchtliche Schwankungen im Krankheitsverlauf bis zum Tode gesehen haben. Wir dürfen ausserdem nicht ausser acht lassen, dass die beiden Tiere aus äusseren Gründen nicht gleichmässig ernährt werden konnten: Tier 261 erhielt kein Wasser zugeführt, hingegen nur den vierten Teil der Uranmenge des Tieres 287, bei ihm hatte sich ein grösserer Niereninfarkt gebildet.

Das anatomische Ergebnis dieser Gruppe ist unerwartet. Von einer Schädigung der Tubuli, so wie sie ron Pohl beschrieben und für kleine Urangaben kennzeichnend genannt wurde, ist kaum etwas zu sehen. Dagegen erfahren durch diese zwei Versuche die Angaben Baehr's ihre volle Bestätigung. Das Uran setzt, in die Nierenarterien eingespritzt, und zwar in Mengen von 0,042 und $0,013 \mathrm{mg}$, eine Schädigung der Glomeruli. Dass diese Schädigung als Entzündung anzusehen sei, dürfte 
keinerlei Widerspruch erfabren. Baehr hat an seinen Tieren nach Einspritzung von $0,8-0,2$ Veränderungen an den Glomerulis gesehen, wie wir sie für die menschliche Glomerulonephritis z. B. nach Scharlach als bezeichnend kennen: Seröse Ausschwitzung in den Kapselraum, Schwellung der Epithelien der Kapsel und der Schlingen, Zellvermehrung an beiden, Halbmondbildung, Polypenbildung, schliesslich Schrumpfung der Glomeruli.

Wir stehen auf Grund unserer Versuche also vor der auffallenden Tatsache: Uran hat in geringen Mengen bei Einspritzung unter die Haut eine tubuläre Entzündung gesetzt, bei Einspritzung in die Nierenarterie eine glomeruläre Entzündung, und zwar handelt es sich nicht um einen Unterschied, der einmal auftrat oder nur von uns beobachtet wurde, sondern regelmässig. Zur Erklärung dieses Untersehiedes in den Befunden muss selbstverständlich in erster Linie die verschiedene Form der Einverleibung verantwortlich gemacht werden. Es kann keinem Zweifel unterworfen sein, dass ein Gift, unter die Haut gespritzt, mindestens mit einer Verdünnung, welche der Blutmenge des Tieres entspricht, wahrscheinlich sogar mit einer unvergleichlich viel höheren Verdünnung in die Niere gelangt, während bei Einspritzung in die Nierenarterie es mit der vollen Stärke (Konzentration), die wir ihm gegeben haben, unmittelbar die Niere und hier in erster Stelle die Glomeruli trifft. Bei Einspritzung von $0,5 \mathrm{ccm} 0,07$ proz. Uran unter die Haut wurde das Uran durch die Vermittelung des allgemeinen Kreislaufes so hochgradig verdünnt, dass es in der Niere nur eine vorübergehende Veränderung setzte, einen krankhaften Reizzustand, eine ${ }_{n}$ Steigerung eines physiologischen Zustandes", wie Baehr es nennt. Bei Einspritzung in die Nierenarterie bleibt die Konzentration des Giltes so stark, dass es eine wirkliche, eine unwiederbringliche Nierenschädigung, eine echte Glomerulonephritis erzeugt (Baehr). Die Art der Nierenveränderung ist abhängig nicht von der einverleibten Giftmenge, sondern in erster Linie von der Konzentration, der Dichte, in welcher das Gift die Niere trifft.

Das Ergebnis der anatomischen Befunde dieser beiden Versuchsreihen lässt sich folgendermassen zusammenfassen: Uran, welches in einer ausserordentlich hohen "biologisehen" Verdünnung die Niere trifft, reizt die Tubuli, und zwar so, dass eine völlige Wiederherstellung möglich ist. Uran in einer Verdünnung, die der Chemiker schon als hoch bezeichnen muss $(1: 160000)$, schädigt beim Eintreffen in die Niere nicht die Tubuli, sondern die Glomeruli; es entsieht eine Urannephritis. Eine vorzügliche Erkrankung dieses oder jenes Nierenabschnittes, wie sie Pohl für die Tubuli angenommen hatte, gibt es nicht. Die Verschiedenheit des Sitzes der Nierenveränderung scheint nach unseren Versuchen lediglich von der Konzentration des Giftes abzuhängen: das dichte Gift schädigt die Glomeruli, das verdünte reizt die Tubuli. Nimmt man die bekannte Tatsache hinzu, dass Uran, in ganz hohen Gaben unter die Haut gespritzt, wie alle Metallsalze Glomeruli und Tubuli schädigt, so scheint der allgemeine Schluss nahe zu liegen, dass es nur eine Sache der Verdünnung eines Giftes sei, aus einem Glomerulusgift ein Tubulus- 
gift, aus einem Schädigungsgift ein Reizgift zu machen. Es wäre sicher lohnend, die Richtigkeit dieses Satzes auch für andere häufig benutzte Gifte zu prüfen.

Abgesehen von diesen anatomischen Befunden und Betrachtungen bleibt noch der klinische Vergleich zwisehen den auf verschiedene Art gespritzten Tieren. Ein grundsätzlicher Unterschied in dem klinischen Ablauf der Erkrankung zwischen den beiden Gruppen besteht nicht, der anatomische Unterschied aber ist offensichtlich. Eine Schädigung des Glomerulusapparates ist als Ursache für das eigenartige Krankheitsbild ('Tod bei offenen Harnwegen) abzulehnen. Denn 1. ist die Veränderung, die wir am Glomerulusapparat feststellen konnten, gering; 2. müsste bei einer Glomerulonephritis Oligurie mit verminderter Stickstoff- und Kochsalzausscheidung auftreten; 3 . hat $\mathrm{Baehr}$ weit erheblichere Veränderungen an den Glomerulis beobachtet, ohne dass die Tiere von selbst zugrunde gingen. Die Glomerulonephritis hat jedenfalls die ausgeschiedenen Mengen nicht unter die Norm herabzudrücken vermocht.

Wir sehen also, dass sowohl bei glomerulärer Schädigung nach Einspritzung in die Nierenarterien als auch bei tubulärer Reizung nach subkutaner Einverleibung die vermehrte Ausscheidung von $\mathrm{NaCl}, \mathrm{N}$ und $\mathrm{H}_{2} \mathrm{O}$ erfolgt, bis schliesslich der Tod eintritt. Die Nierenveräıderungen an sich können, soweit es sich anatomisch beurteilen lässt, nicht Todesursache sein, dazu sind sie verhältnismässig zu geringfügig. Eine Erklärung für die erhöhte Nierentätigkeit ist gegeben, wenn wir dem Uran in bestimmten Dosen die Eigenschaft zusprechen, die Niere zur Hyperfunktion anzuregen, wie es Dünner in seiner ersten Arbeit getan hat. Dünner glaubte zu dieser Annahme berechtigt zu sein, da er das klinische Bild der offenen Harnwege auch nach direkter Einspritzung in die Nierenarterie beobachtete, in der das Gift bleiben sollte, ohne sonst in den Kreisiauf zu gelangen. Man muss sich nur fragen, warum nicht die Tiere, wenn ihre Nieren dem Organismus $\mathrm{NaCl}, \mathbf{N}$ und $\mathrm{H}_{2} \mathrm{O}$ entziehen, den entstandenen Schaden durch stärkeres Fressen zu kompensieren suchen. Statt dessen nehmen sie weniger Nahrung zu sich. Diese Tatsache weckt den Verdacht, ob nicht doch bei der intraarteriellen Einspritzung Uran in den Körper gelangen kann. Dünner hatte allerdings gerade von dieser Methode erhofft, dass das Uran nicht in den Kreislauf gelangen könne. $D$ ünner's Versuche erscheinen auf Grund unserer neueren Erfahrungen in einem anderen Lichte. Aus intraarteriellen Versuchen Baehr's in eine Niere ersieht man, dass später die andere Niere tubuläre Veränderungen zeigen kann; es muss also hierhin Uran gekommen sein. Und wenn es Dünner gelungen ist, nach Einspritzung in eine Niere und Exstirpation dieser Niere nach 2 Stunden die Tiere vollkommen gesund zu erhalten, so schliesst das nicht aus, dass in den anderen Fällen allmählich Uran aus dem Nierendepot in andere Körperteile gelangen kann.

Unbeschadet dieser Einwände bleibt die Tatsache, dass die Niere bei kleinen Urangaben stark arbeitet, gleichgültig, ob das Uran je nach der Art der Verabreichung mehr den Glomerulus- oder den Tubulusapparat angreift, d. h. also: die Hyperfunktion, wie $\mathrm{Pohl}$ angenommen hatte, ist nicht abhängig von einer bestimmten Erkrankung der Niere. Man könnte 
392 Dünner u. Siegfried, Experiment. u. patholog.-anatom. Untersuchungen usw.

sich sogar so ausdrücken, dass die Niere trotz der pathologischen Veränderungen sehr gut funktioniert. Nimmt man diesen Standpunkt ein, so darf man von einer elektiven Wirkung des Urans auf die Nierenfunktion sprechen. Dabei müssen wir die Möglichkeit der übrigen Körperschädigung durch das Uran zugeben.

Zum Beweise dieser Annahme wäre erforderlich gewesen, an den übrigen Organen anatomische Veränderungen aufzuzeigen. Leider sind wir nachträglich nicht in der Lage gewesen, diese Forderungen zu erfüllen, da wir nur die Nieren für mikroskopische Untersuchung aufgehoben hatten.

\section{Zusammenfassung.}

Nach subkutaner Einspritzung und direkter Injektion sehr kleiner Urandosen in die Nierenarterien erfolgt beim Kaninchen eine mehr oder weniger starke Ausschwemmung von $\mathrm{N}, \mathrm{NaCl}$ und Wasser, unter der das Tier schliesslich sterben kann.

Anatomisch findet man nach der subkutanen Einspritzung an den Nieren nicht eine Ausschwemmung des Tubulusepithels (Pohl), sondern nur eine starke Abplattung bei höchster Erweiterung der Tubuli (tubuläre Reizung). Diese Veränderung kann reparabel sein.

Bei den Injektionen in die Niere direkt beobachtet man eine Ent$z$ ündung an den Glomerulis (Glomerulonephritis).

Der auffällige klinische Verlauf (Hyperfunktion der Niere) ist durch die Nierenveränderung nicht erklärt. Anscheinend spielt eine elektive Wirkung des Urans auf die Nierentätigkeit dabei eine besondere Rolle. Das schliesst nicht unbedingt aus, dass daneben eine Vergiftung anderer Organe durch das Uran erfolgt. 